\title{
Evidence of Stage Shift in Women Diagnosed With Ovarian Cancer During Phase II of the United Kingdom Familial Ovarian Cancer Screening Study
}

\section{Citation}

Rosenthal, A. N., L. S. Fraser, S. Philpott, R. Manchanda, M. Burnell, P. Badman, R. Hadwin, et al. 2017. "Evidence of Stage Shift in Women Diagnosed With Ovarian Cancer During Phase II of the United Kingdom Familial Ovarian Cancer Screening Study." Journal of Clinical Oncology 35 (13): 1411-1420. doi:10.1200/JC0.2016.69.9330. http://dx.doi.org/10.1200/JC0.2016.69.9330.

\section{Published Version}

doi:10.1200/JC0.2016.69.9330

\section{Permanent link}

http://nrs.harvard.edu/urn-3:HUL.InstRepos:35014367

\section{Terms of Use}

This article was downloaded from Harvard University's DASH repository, and is made available under the terms and conditions applicable to Other Posted Material, as set forth at http:// nrs.harvard.edu/urn-3:HUL.InstRepos:dash.current.terms-of-use\#LAA

\section{Share Your Story}

The Harvard community has made this article openly available.

Please share how this access benefits you. Submit a story.

Accessibility 


\section{Evidence of Stage Shift in Women Diagnosed With Ovarian Cancer During Phase II of the United Kingdom Familial Ovarian Cancer Screening Study}

Adam N. Rosenthal, Lindsay S.M. Fraser, Susan Philpott, Ranjit Manchanda, Matthew Burnell, Philip Badman, Richard Hadwin, Ivana Rizzuto, Elizabeth Benjamin, Naveena Singh, D. Gareth Evans, Diana M. Eccles, Andy Ryan, Robert Liston, Anne Dawnay, Jeremy Ford, Richard Gunu, James Mackay, Steven J. Skates, Usha Menon, and Ian J. Jacobs on behalf of the United Kingdom Familial Ovarian Cancer Screening Study collaborators

Author affiliations and support information (if applicable) appear at the end of this article.

Published at jco.org on February 27, 2017

A.N.R. and L.S.M.F. contributed equally to this study. U.M. and I.J.J. contributed equally to this study.

This study reports about off-license use of cancer antigen 125 and sonography for screening for ovarian cancer in

a registered clinical trial.

Clinical trial information: ISRCTN32794457

Corresponding author: Ian J. Jacobs, MD University of New South Wales Australia, Sydney, New South Wales 2052

Australia; e-mail: i.jacobs@unsw.edu.au.

(C) 2017 by American Society of Clinical Oncology. Licensed under the Creative Commons Attribution 4.0 License.

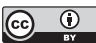

0732-183X/17/3513w-1411w/\$20.00
ASSOCIATED CONTENT

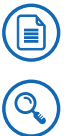

See accompanying Editorial

on page 1384

Data Supplement

DOI: $10.1200 / J C O .2016 .69 .9330$

DOI: $10.1200 / J C O .2016 .69 .9330$

\section{$\begin{array}{llllllll}\text { A } & \text { B } & \mathbf{S} & \mathbf{T} & \mathbf{R} & \mathbf{A} & \mathbf{C} & \mathbf{T}\end{array}$}

\section{Purpose}

To establish the performance of screening with serum cancer antigen 125 (CA-125), interpreted using the risk of ovarian cancer algorithm (ROCA), and transvaginal sonography (TVS) for women at high risk of ovarian cancer (OC) or fallopian tube cancer (FTC).

\section{Patients and Methods}

Women whose estimated lifetime risk of OC/FTC was $\geq 10 \%$ were recruited at 42 centers in the United Kingdom and underwent ROCA screening every 4 months. TVS occurred annually if ROCA results were normal or within 2 months of an abnormal ROCA result. Risk-reducing salpingooophorectomy (RRSO) was encouraged throughout the study. Participants were observed via cancer registries, questionnaires, and notification by centers. Performance was calculated after censoring 365 days after prior screen, with modeling of occult cancers detected at RRSO

\section{Results}

Between June 14, 2007, and May 15, 2012, 4,348 women underwent 13,728 women-years of screening. The median follow-up time was 4.8 years. Nineteen patients were diagnosed with invasive OC/FTC within 1 year of prior screening (13 diagnoses were screen-detected and six were occult at RRSO). No symptomatic interval cancers occurred. Ten (52.6\%) of the total 19 diagnoses were stage I to II OC/FTC (Cl, 28.9\% to $75.6 \%)$. Of the 13 screen-detected cancers, five (38.5\%) were stage I to II $(\mathrm{Cl}, 13.9 \%$ to $68.4 \%)$. Of the six occult cancers, five (83.3\%) were stage I to $\mathrm{II}(\mathrm{Cl}, 35.9 \%$ to $99.6 \%)$. Modeled sensitivity, positive predictive value, and negative predictive value for OC/FTC detection within 1 year were $94.7 \%(\mathrm{Cl}, 74.0 \%$ to $99.9 \%), 10.8 \%(6.5 \%$ to $16.5 \%)$, and $100 \%(\mathrm{Cl}, 100 \%$ to $100 \%)$, respectively. Seven (36.8\%) of the 19 cancers diagnosed $<1$ year after prior screen were stage IIIb to IV (Cl, 16.3\% to 61.6\%) compared with 17 (94.4\%) of 18 cancers diagnosed $>1$ year after screening ended $(\mathrm{Cl}, 72.7 \%$ to $99.9 \% ; P<.001)$. Eighteen $(94.8 \%)$ of 19 cancers diagnosed $<1$ year after prior screen had zero residual disease (with lower surgical complexity, $P=.16)(\mathrm{Cl}, 74.0 \%$ to $99.9 \%)$ compared with $13(72.2 \%)$ of 18 cancers subsequently diagnosed $(\mathrm{Cl}, 46.5 \%$ to $90.3 \% ; P=.09)$.

\section{Conclusion}

ROCA-based screening is an option for women at high risk of OC/FTC who defer or decline RRSO, given its high sensitivity and significant stage shift. However, it remains unknown whether this strategy would improve survival in screened high-risk women.

J Clin Oncol 35:1411-1420. (c) 2017 by American Society of Clinical Oncology. Licensed under the Creative Commons Attribution 4.0 License: http://creativecommons.org/licenses/by/4.0/

\section{INTRODUCTION}

Inherited mutations in BRCA1 and BRCA2 and Lynch syndrome (LS) account for a significant minority ( $15 \%$ to $25 \%$ ) of ovarian cancers (OCs) ${ }^{1,2}$ and confer a high risk for OC: $11 \%$ to $37 \%$ by age
70 years in BRCA2 carriers and $39 \%$ to $65 \%$ in BRCA1 carriers. ${ }^{3,4}$ Other lower-penetrance homologous repair pathway genes have been implicated in familial OC. ${ }^{5,6}$

Although medium-term survival with $B R C A$ associated OC exceeds that of sporadic OC, ${ }^{7,8}$ the long-term outlook remains poor. ${ }^{9}$ Risk-reducing 
salpingo-oophorectomy (RRSO) for women older than 35 years of age to prevent OC or fallopian tube cancer (FTC) and to detect occult neoplasia is recommended as the only proven mortalityreducing intervention. ${ }^{10,11}$ Although effective when used premenopausally, ${ }^{10,11}$ RRSO causes infertility and premature menopause, with associated cardiovascular risks, ${ }^{12}$ osteoporosis, ${ }^{13}$ and neurologic risks ${ }^{14}$ (although premature menopause can be treated with hormone replacement therapy). Some women decline RRSO regardless of OC risk, and others prefer to defer it (eg, until menopause). Effective OC screening would be a welcome option for such women.

Annual OC screening in the general population that uses a cutoff for the serum tumor marker cancer antigen 125 (CA-125) was associated with improved survival. ${ }^{15}$ In the high-risk population, we $\mathrm{e}^{16}$ and others ${ }^{17-20}$ have reported annual screening using a CA-125 cutoff and transvaginal sonography (TVS). Although we demonstrated high sensitivity $(>80 \%)$ and positive predictive value (PPV; 25\%), two symptomatic interval cancers occurred, and $69 \%$ of detected cancers were stage III to IV. ${ }^{16}$ This annual screening interval has been associated with a poor 10 -year survival rate of $36 \%$ in $B R C A 1 / 2$ carriers. $^{21}$

Multimodal screening with the risk of ovarian cancer algorithm (ROCA) to interpret serial CA-125 results, and TVS as a second-line test, in the randomized general-population United Kingdom Collaborative Trial of Ovarian Cancer Screening (UKCTOCS) achieved high sensitivity and specificity. ${ }^{22,23}$ Significantly more $(40 \%)$ low-volume (stages I, II, or IIIa) invasive epithelial ovarian/peritoneal cancers were identified compared with unscreened controls $(26 \%)$ on an intention-to-screen analysis, and the trial provided an encouraging, though not definitive, mortality benefit. ${ }^{24}$

Random assignment to a nonscreening arm is thought to be unacceptable to high-risk women and clinicians. ${ }^{16}$ Even if ethical, it would likely be unfeasible, so research screening in this population is limited to prospective cohort studies. To our knowledge, this is the first published study to use ROCA-based screening to define sensitivity in the high-risk population.

\section{PATIENTS AND METHODS}

A prospective multicenter cohort screening study was undertaken within the United Kingdom (UK) National Health Service (NHS). Ethical approval was given by the Eastern Multicentre Research Ethics Committee (Reference No. 97/5/007). The protocol can be found online. ${ }^{25}$

\section{Entry Criteria}

High-risk women at an estimated minimum $10 \%$ lifetime risk of OC were recruited; inclusion criteria (Data Supplement, online only) depended on family history or predisposing mutations. Documentation (death certificates and/or histopathology reports) of relevant cancers was required, and eligibility was confirmed by the coordinating center (CC). Clinical genetic testing was performed by accredited NHS laboratories. After screening ended, $45.2 \%$ of the study population underwent BRCA1/2 next-generation sequencing research testing. ${ }^{26}$

\section{Recruitment}

Participants were recruited at 42 centers in the UK by specialist nurses, clinical geneticists, or gynecologists. In December 2006, participants in the UK Familial Ovarian Cancer Screening Study (UK FOCSS) Phase I (annual screening using a CA-125 cutoff and TVS) ${ }^{16}$ were invited to join this study-UK FOCSS Phase II. Other participants were recruited de novo. Women were counseled about RRSO and screening limitations. All participants provided written consent.

\section{Screening}

The trial database ${ }^{16}$ scheduled serum CA-125 tests every 4 months and TVS annually. Venipuncture packs were mailed to participants for use in primary care and blood samples returned to the CC laboratory for CA125 assay. ${ }^{23}$ Results were uploaded to the database, which calculated OC risk using the high-risk ROCA, which also incorporated the higher a priori risk in our population and different reference levels for risk stratification for postmenopausal compared with premenopausal women, because of the higher baseline CA-125 and variability in premenopausal women. ${ }^{27}$ Menopausal status was determined by the database by using the age of participants and their responses to questions about gynecologic history and/or symptoms, which were returned with serum samples (Data Supplement).

Initial risk of ovarian cancer (ROC) was based on initial CA-125 level and estimated age-specific OC incidence. Subsequently, ROC was based on absolute CA-125 level and rate of change. Initially high or increasing CA125 levels (even $<30 \mathrm{iU} / \mathrm{ml}$ ) generated a high ROC, whereas initially low, stable-high (even $>30 \mathrm{iU} / \mathrm{ml}$ ), or decreasing levels generated low ROCs. ROCA results were used for triage, including expedition of repeat CA-125 tests and/or TVS after abnormal results (Data Supplement).

Collaborating centers performed scans and completed proformas (Data Supplement), which were classified by the database according to predetermined criteria (Data Supplement). ${ }^{16}$ When indicated, women were referred to a gynecologist for clinical assessment, with a view to surgical removal of the fallopian tubes and ovaries. The final decision about surgery was made after additional investigation and discussion with the patient.

\section{Follow-Up}

Participants were flagged (by their unique NHS number) with relevant cancer registries, which provided cancer and/or death data. ${ }^{16} \mathrm{Col}-$ laborators notified the $\mathrm{CC}$ when women withdrew before routine screening ended (June 30, 2011). Women were observed through cancer registries with censorship that was based on date of death, last notification from the registry, or last contact if they were lost to registry follow-up. Participants were sent health questionnaires in January 2011 and April 2013 specifically asking about surgery that involved removal of fallopian tubes/ovaries and cancer diagnosis.

\section{Diagnostic Documentation}

Whenever women underwent salpingo-oophorectomy, the $\mathrm{CC}$ obtained documentation of indication, operation notes, and histopathology/ cytopathology reports. These were reviewed by a gynecologic oncologist (A.N.R.) and pathologist (E.B./N.S.) and were classified according to the International Statistical Classification of Diseases and Related Health Problems, 10th revision (ICD-10). Trial surgery was defined as either screen-positive or screen-related (nonconcerning abnormal results, such as simple cysts and/or transient/stable abnormal ROC results that contributed to the participant's decision to undergo surgery). ${ }^{15}$ Centers were provided with an RRSO protocol, which advocated serial sectioning of fallopian tubes/ovaries (Data Supplement). A surgical complexity score was assigned using recognized criteria (Data Supplement). ${ }^{28}$

\section{Statistical Analysis}

For performance analyses, data were censored 365 days after the last UK FOCSS screen. Invasive OC, FTC, or primary peritoneal cancer (PPC) diagnosed $<365$ days after the last screen were included. Cancers that occurred after censoring and diagnosed before February 28, 2016 were 
reported but not included in the performance analyses. The study was powered to estimate sensitivity within 10\% (expected 95\% CI), given an annual OC incidence of $0.5 \%$. Analyses were done with Stata (version 14; STATA, College Station, TX).

Compliance with blood tests and scans was defined as the proportion of requested tests received by the CC. These were analyzed separately and according to screen type (eg, routine, protocol-indicated repeat).

Women who underwent salpingo-oophorectomy were only classified as having undergone RRSO if they were asymptomatic, they had normal results at prior screen, and the recruiting center indicated RRSO as the reason for withdrawal. Cases in which abnormal results prompted surgery were true positive (TP) if invasive epithelial OC/FTC was diagnosed. All other diagnoses (including borderline/benign tumors) that resulted from surgery that was prompted by abnormal results were false positive (FP). Cases in which nonconcerning test results (simple cysts/transiently elevated CA-125) contributed to the decision for surgery were classified as screen-related surgery, to provide estimates of likely additional surgeries in any future screening program. True-negative (TN) designations were for those patients in whom the last screen was normal and no OC/FTC was diagnosed $<365$ days. Patients who presented with clinically diagnosed interval cancers between screens or $<365$ days after the final screen were considered false negative (FN). Prevalent cases were those diagnosed at first screen. Incident cases were those diagnosed subsequently. For women who transferred from Phase I (annual CA-125 cutoff and scan) to Phase II (ROCA every 4 months and annual TVS), their first Phase II screen was classified as incident.

We reported performance according to whether occult cancers diagnosed $<365$ days after a prior screen were classified as FN or TP. ${ }^{16}$ In an attempt to estimate true sensitivity, we assumed that the proportion of occult cancers identified at RRSO, which would have been screen detected had women not undergone surgery, would be identical to that observed in those who continued screening. We then used the lower confidence limit of

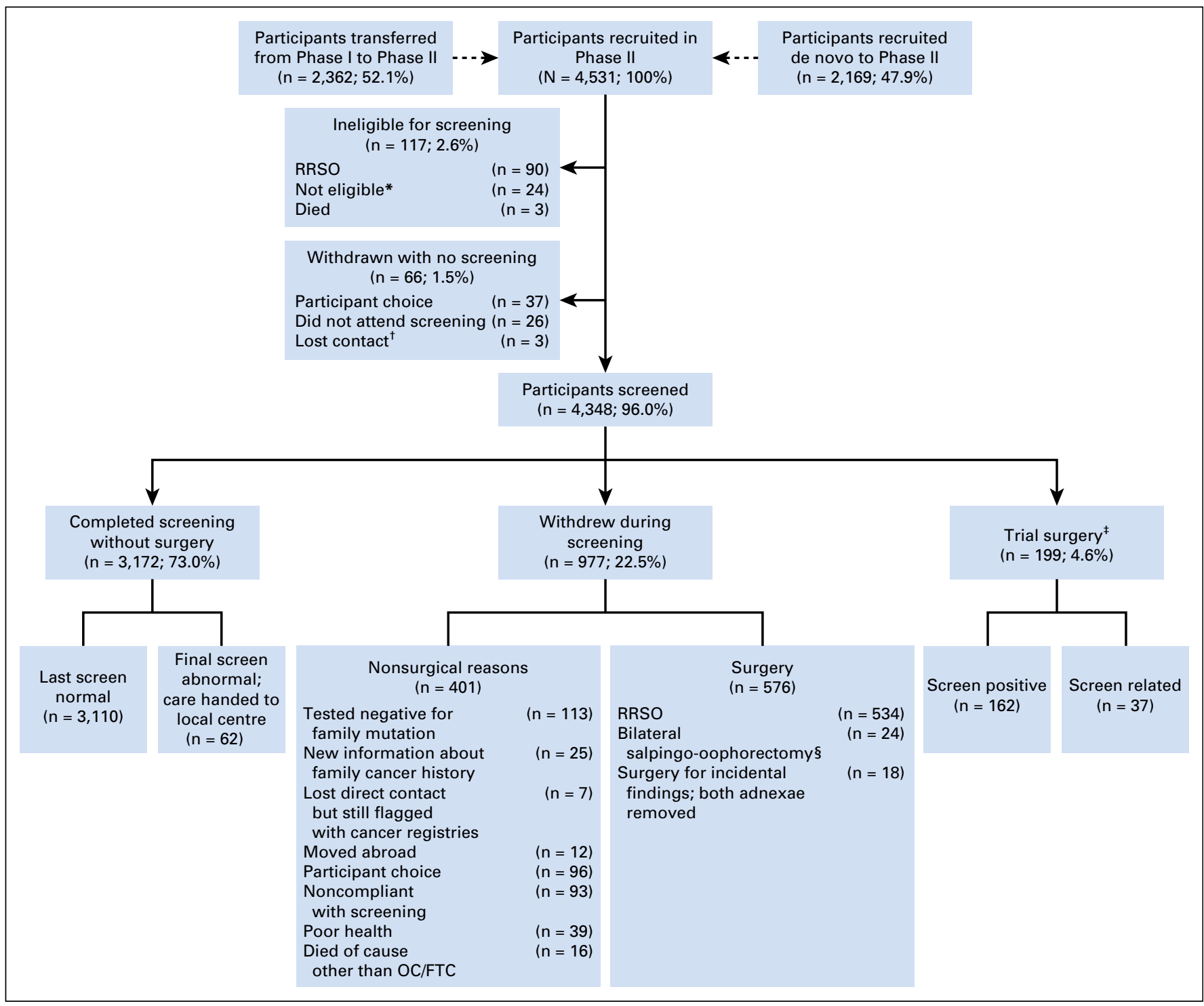

Fig 1. CONSORT diagram. Percentages refer to the proportion of the total in preceding box. $\left(^{*}\right)$ Ineligible due to new information about family cancer history, tested

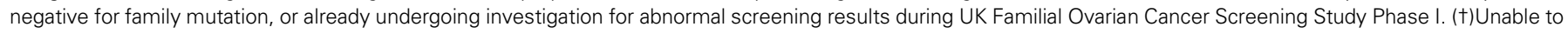

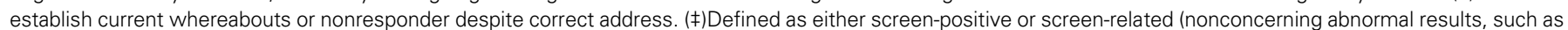

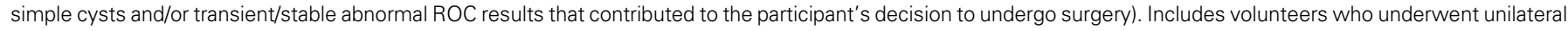

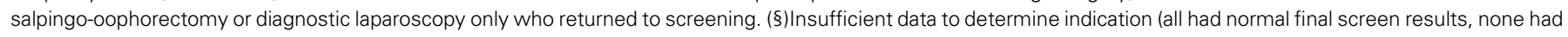
cancer). RRSO, risk-reducing salpingo-oophorectomy. 
observed sensitivity in women who did not undergo RRSO as a conservative estimate of occult cancer detection sensitivity, and we rounded the predicted number of occult cancers detected to the nearest integer.

Because the protocol required parallel CA-125 and TVS, the results of which influenced each other's timing, it was not possible to calculate performance characteristics per test. Therefore, we calculated these metrics per woman-screen year (WSY) for the protocol overall.

To allocate WSYs to correct outcomes we applied the following rules; for TP and FP detection screens, the WSY that commenced with that screen was classified as TP or FP, respectively. WSYs before the detection screen were TN. For occult cancers diagnosed $<365$ days after prior screen, the WSY that commenced with that screen was classified as FN or TP (dependent on analysis type), and prior WSYs were TN. For TN cases, all WSYs were classified TN.

To investigate potentially avoidable delays, we analyzed screening and screen-to-surgery intervals. ${ }^{16}$ Detection screens were defined as an abnormal TVS and/or abnormal ROC that led to a surgery/biopsy that diagnosed OC/FTC. Delayed screens were defined as any detection screen performed after the protocol-indicated date. Delay was calculated as the detection screen date minus the protocol-indicated date. The interval from screen date to diagnosis was calculated to the date of surgery/biopsy. We compared International Federation of Gynecology and Obstetrics stage and postsurgery zero residual disease rates in OC/ FTC diagnosed during and $<365$ days from the end of UK FOCSS screening with those diagnosed $>365$ days after screening ended. We also compared stage-distribution and zero residual disease rates in incident screen-detected cancers in Phases I and II of the study. No survival analysis was performed because of the low number of events observed.

\section{RESULTS}

Between June 14, 2007, and May 15, 2012, 4,531 women were recruited. This included 2,362 (66.3\%) of 3,563 eligible women from UK FOCSS Phase I (Fig 1). Table 1 lists inclusion indications. A total of $183(4.0 \%)$ women withdrew before screening (Fig 1). The outcome of the remaining 4,348 women (96.0\%) were analyzed. The median age at recruitment was 45.5 years (range, 34.2 to 84.8 years). Of the eligible women, 1,278 women $(29.4 \%$ of participants) underwent mutation testing, and 1,965 (45.2\%) subsequently underwent next-generation sequencing. ${ }^{24}$ Overall, $924(21.3 \%)$ women were known mutation carriers (further demographics in Data Supplement).

The last cancer notifications from NHS Digital were received on February 28, 2016 (England/Wales), May 15, 2016 (Scotland), and April 19, 2016 (Northern Ireland); the last death notifications were received on March 14, 2016 (all countries). Follow-up was possible for 4,046 women (93.1\%). Median follow-up beyond last screen/withdrawal was 4.7 years (range, 0 to 8.7 years).

\section{Screening/Compliance}

The 4,348 screened participants underwent 13,728 WSY (median, 3.26 screen-years per woman; range, 1.00 to 5.94 screenyears per woman). A total of 189 women $(4.3 \%)$ ceased screening by choice. Five hundred fifty-eight $(12.8 \%)$ ceased because of surgical removal of both fallopian tubes/ovaries for RRSO $(\mathrm{n}=534)$ or indeterminate reasons $(\mathrm{n}=24)$. A total of 377 women $(8.7 \%)$ whose last screen was abnormal continued screening until May 15, 2012, by which time 315 had normal results and did not
Table 1. Inclusion Criteria and Mutation Status in Screened Participants $(\mathrm{N}=4,348)$

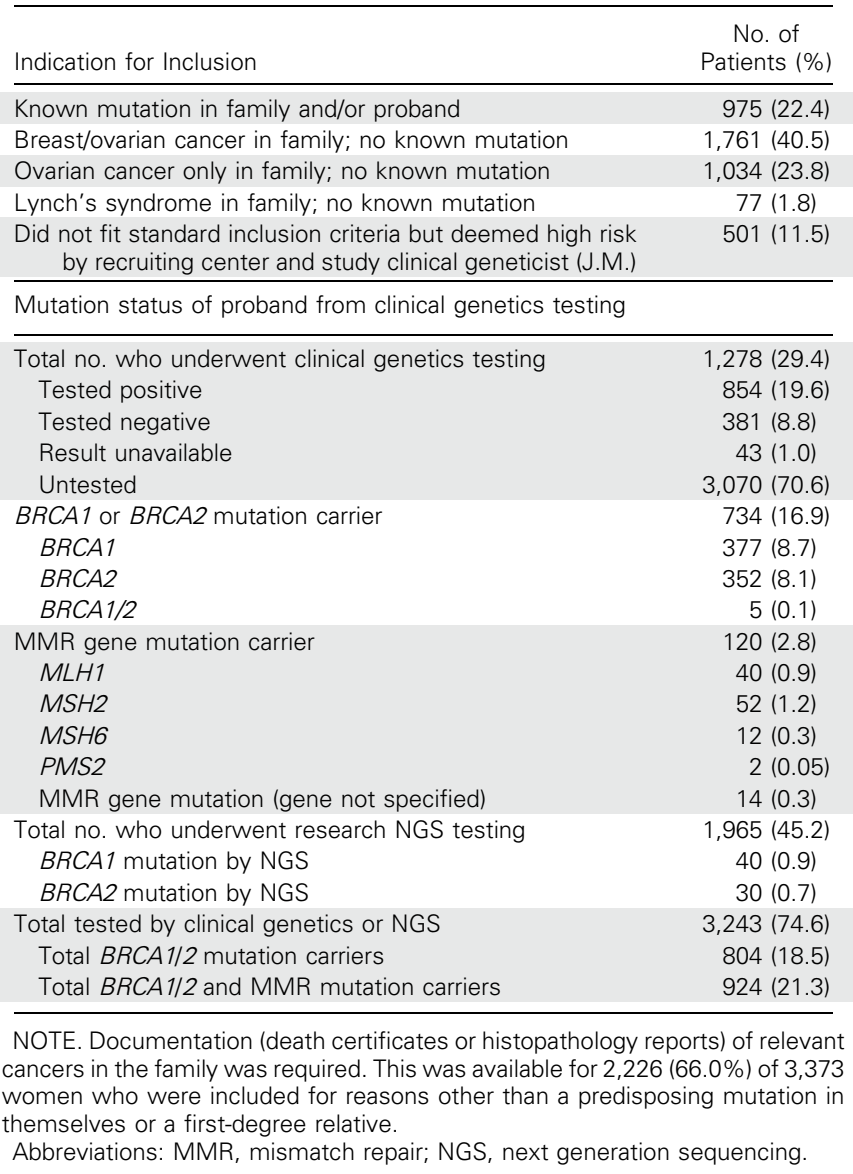

undergo surgery. Care was transferred to local gynecologists for the 62 women who still had abnormal results. Three of these 62 women underwent surgery; none had cancer.

Compliance with requested routine CA-125 tests and scans were $92.1 \%(27,138$ of 29,450 CA-125 tests) and $94.6 \%(9,100$ of 9,619 scans), respectively (Data Supplement). Compliance for scans was based on reports received, not scans undertaken, so it is likely an underestimate. Protocol-indicated repeat test compliance was higher: $97.4 \%(4,716$ of 4,834$)$ of blood tests, and $98.8 \%(2,792$ of 2,825 ) of scans requested were received.

Of the 32,587 blood samples received, routine tests comprised $83.3 \%(27,138$ of 32,587$)$, protocol-triggered repeats comprised $14.5 \%(4,716$ of 32,587$)$, and $2.2 \%$ (733 of 32,587$)$ were requested by study clinicians (eg, because CA-125 levels had increased by $\geq 50 \%$, despite a normal ROC). A total of 2,233 (6.9\%) of 32,587 blood samples were discarded because they arrived more than 56 hours after venipuncture. Of the 12,038 scan results, $75.6 \%(9,100$ of $12,038)$ were annual, $23.2 \%$ (2,792 of 12,038$)$ were triggered early by abnormal ROC results and/or previous abnormal scans, and 1.2\% (146 of 12,038) were repeated because of a poor view of the ovaries.

Overall, $162(3.7 \%)$ of 4,348 women underwent screen-positive trial surgery. Thirteen of these 162 women had screen-detected cancers. The remaining $149(3.4 \%)$ of the 4,348 women underwent 
false-positive surgery prompted by abnormal results (Table 2). Of these 149 women who underwent false-positive surgery, 46 (30.9\%) had an abnormal ROC alone, 62 (41.6\%) had an abnormal scan alone, and $41(27.5 \%)$ had abnormal results for both tests. Overall, 95 $(63.8 \%)$ of the 149 women who underwent false-positive surgery had benign ovarian pathology, two (1.3\%) had borderline ovarian tumors, and $52(35.0 \%)$ had other/no pathology identified. An additional 37 $(0.9 \%)$ of the 4,348 women underwent screen-related trial surgery.

\section{Invasive OC/FTC/PPC}

Thirty-seven women were diagnosed with invasive cancer before February 28, 2016 (Table 3); nineteen occurred during 13,728 WSY $<365$ days after prior screen and/or withdrawal (annual incidence $0.14 \%$ ). In addition, 18 women were diagnosed $>365$ days after their last UK FOCSS screen (median, 666 days; range, 400 to 2,159 days). The median age at diagnosis in the 37 women diagnosed with OC/FTC/PPC was 50 years (range, 37 to 79 years). All diagnoses occurred in families with hereditary breast-ovarian cancer. Thirty-four $(91.2 \%)$ of the 37 women were diagnosed with high-grade serous carcinoma. Cancers in 31 $(83.8 \%)$ of the 37 women occurred in mutation carriers-24 (64.9\%) were BRCA1 carriers and seven (18.9\%) were BRCA2 carriers. Three $(8.1 \%)$ of the 37 women were $B R C A 1 / 2$ negative; one $(2.7 \%)$ of the 37 women had a BRCA2 variant of unknown significance; two $(5.4 \%)$ of the 37 women were untested. Of the 37 women diagnosed with OC/FT/PPC, 23 (62.2\%) knew they carried pathogenic mutations and $14(37.8 \%)$ had a history of breast cancer. No OC occurred in women with a family history of LS or those who were mutation carriers for the syndrome ( $\mathrm{n}=192 ; 558$ WSY).

The 19 invasive OC/FTCs diagnosed within 365 days of prior screen included one prevalent screen-positive OC (International Federation of Gynecology and Obstetrics stage IIIc) and 18 incident cancers. Twelve of the 18 incident OC/FTCs were screen detected and six were occult cancers identified at RRSO. Of the 12 patients with incident screen-detected cancer, $11(91.7 \%)$ had an abnormal ROC and $5(41.7 \%)$ had a normal TVS at detection (compared with zero of 13 patients who had normal TVS at detection in UK FOCSS Phase I; $P=.015$ ). The median CA-125 level at detection was $53.8 \mathrm{iU} / \mathrm{ml}$ (range, 11.7 to $219.2 \mathrm{iU} / \mathrm{ml}$ ) in UK FOCSS Phase II ( $<30 \mathrm{iU} / \mathrm{ml}$ in four of 12 patients) compared with $73 \mathrm{iU} / \mathrm{ml}$ (range, 4 to $3,874 \mathrm{iU} / \mathrm{ml}$ ) in Phase I, which did not mandate assay type and recommended premenopausal and postmenopausal cutoffs of 35 and $30 \mathrm{iU} / \mathrm{ml}$, respectively, rather than according to the ROCA. Five $(38.5 \%)$ of the 13 screendetected OC/FTCs (CI, $13.9 \%$ to $68.4 \%)$ and $5(83.3 \%)$ of the six occult OC/FTCs (CI, 35.9\% to $99.6 \%$ ) were stage I to II. Overall, $10(52.6 \%)$ of the 19 cancers diagnosed within 365 days of prior screen were stage I to II (CI, $28.9 \%$ to $75.6 \%$ ).

Eighteen cancers were diagnosed $>365$ days after the end of UK FOCSS screening. Two occult cancers were detected at RRSO, three cancers were detected at annual screening performed locally, and 13 were detected when women presented with symptoms. Only one $(5.6 \%)$ of the 18 cancers was diagnosed at stage I to II (CI, $0.2 \%$ to $27.3 \%)$.

Women were significantly less likely to be diagnosed with stage IIIb to IV OC during UK FOCSS Phase II screening (seven [36.8\%] of $19 ; \mathrm{CI}, 16.3 \%$ to $61.6 \%$ ) compared with those diagnosed subsequently (17 [94.4\%] of 18 ; CI, $72.7 \%$ to $99.9 \%$; $P<.001$ ). Twelve $(92.3 \%)$ of 13 women who had screen-detected cancers had zero postsurgical residual disease (CI; 64.0\% to 99.8\%). Overall, 18 $(94.8 \%)$ of 19 women diagnosed with OC during UK FOCSS had zero postsurgical residual (CI, 74.0\% to $99.9 \%$ ) compared with 13 (72.2\%) of 18 women who were diagnosed subsequently (CI, $46.5 \%$ to $90.3 \% ; P=.09$ ). None of the women diagnosed during UK FOCSS required complex surgery, one had interval surgery. Three of the subsequently diagnosed women required complex surgery, seven had interval surgery, and two had no debulking (Table 3). The proportion of women diagnosed with OC during UK FOCSS who had neoadjuvant chemotherapy (1 [5.3\%] of 19 women; CI, $0.1 \%$ to $26.0 \%$ ) was significantly lower than in the women diagnosed subsequently

Table 2. Diagnoses in Women Who Underwent False-Positive Surgery to Rule Out Ovarian Cancer As a Result of Abnormal Screening Tests ( $n=149$ )

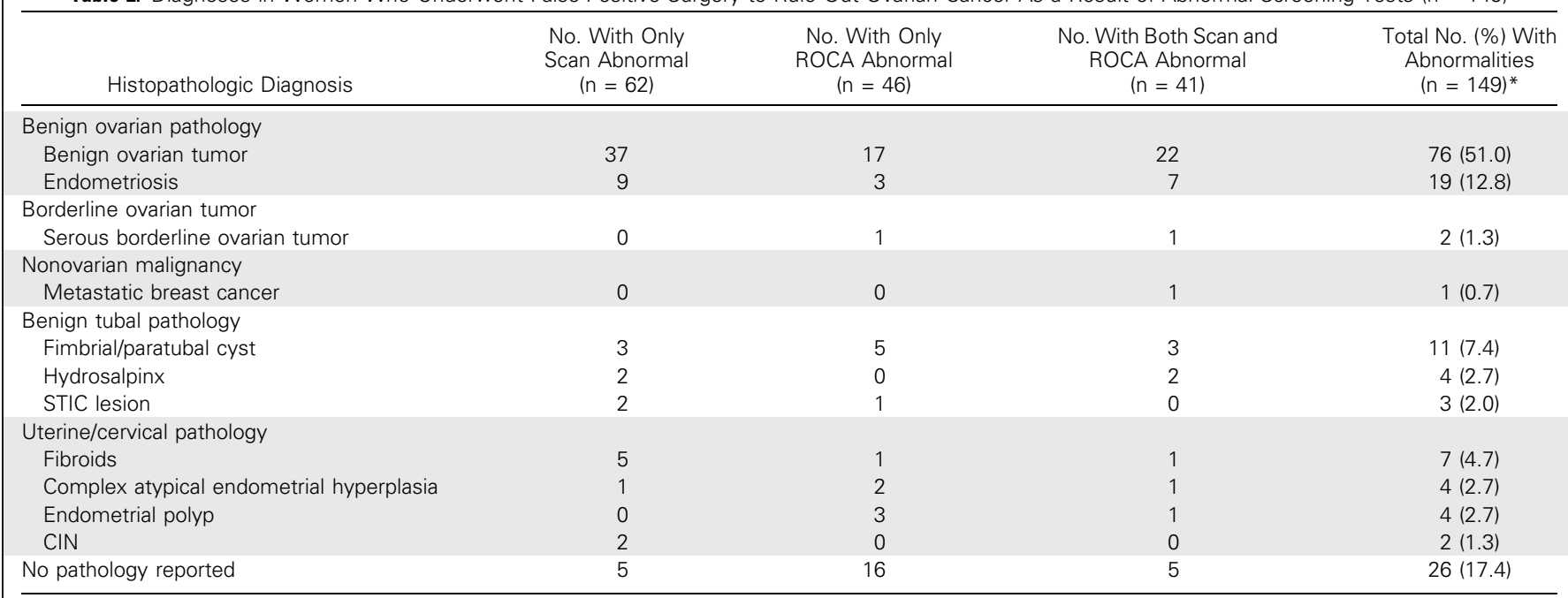

Abbreviations: CIN, cervical intraepithelial neoplasia; ROCA, risk of ovarian cancer algorithm; STIC, serous tubal intraepithelial carcinoma.

*Total of 159 diagnoses in 149 women, because some women had more than one diagnosis. 


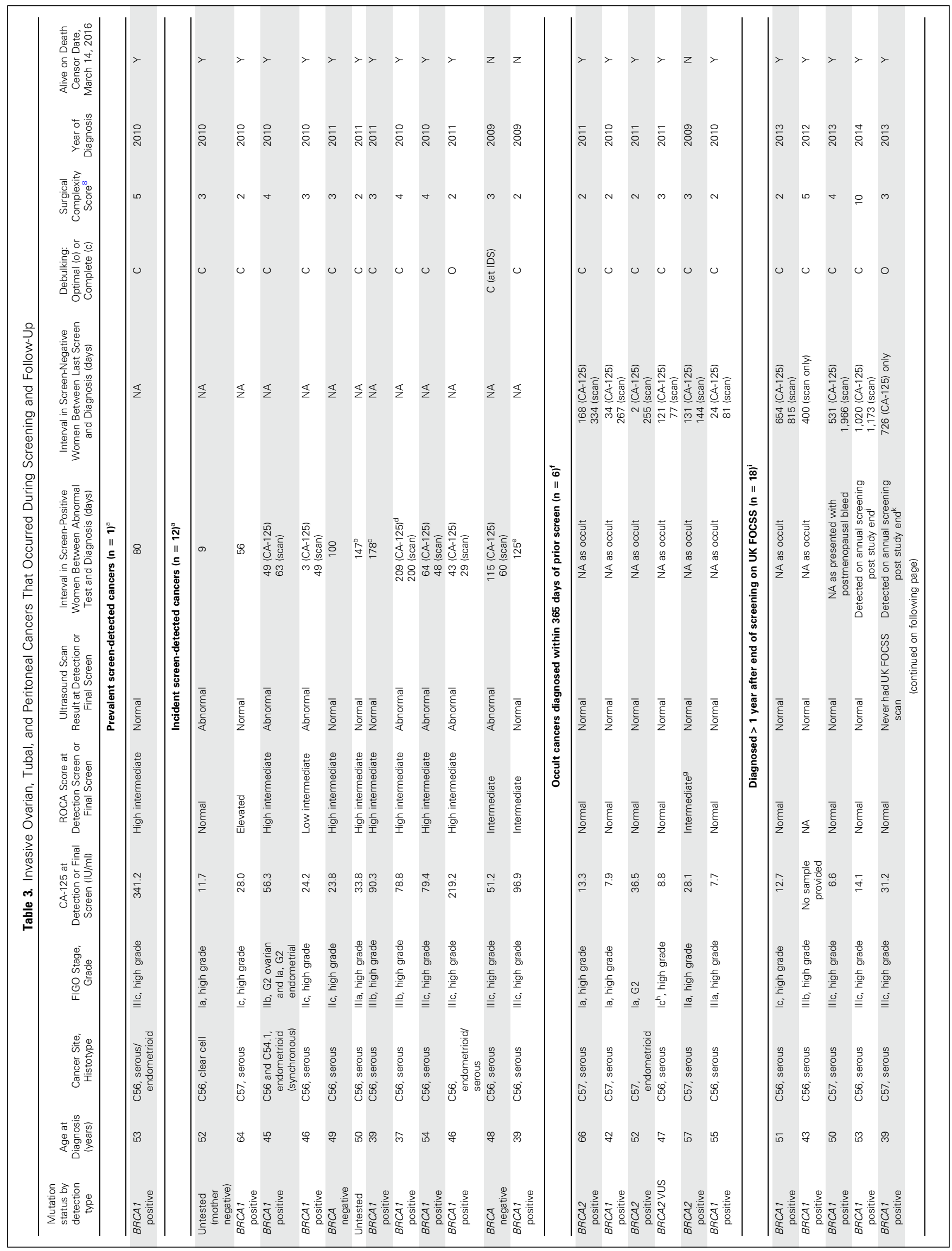




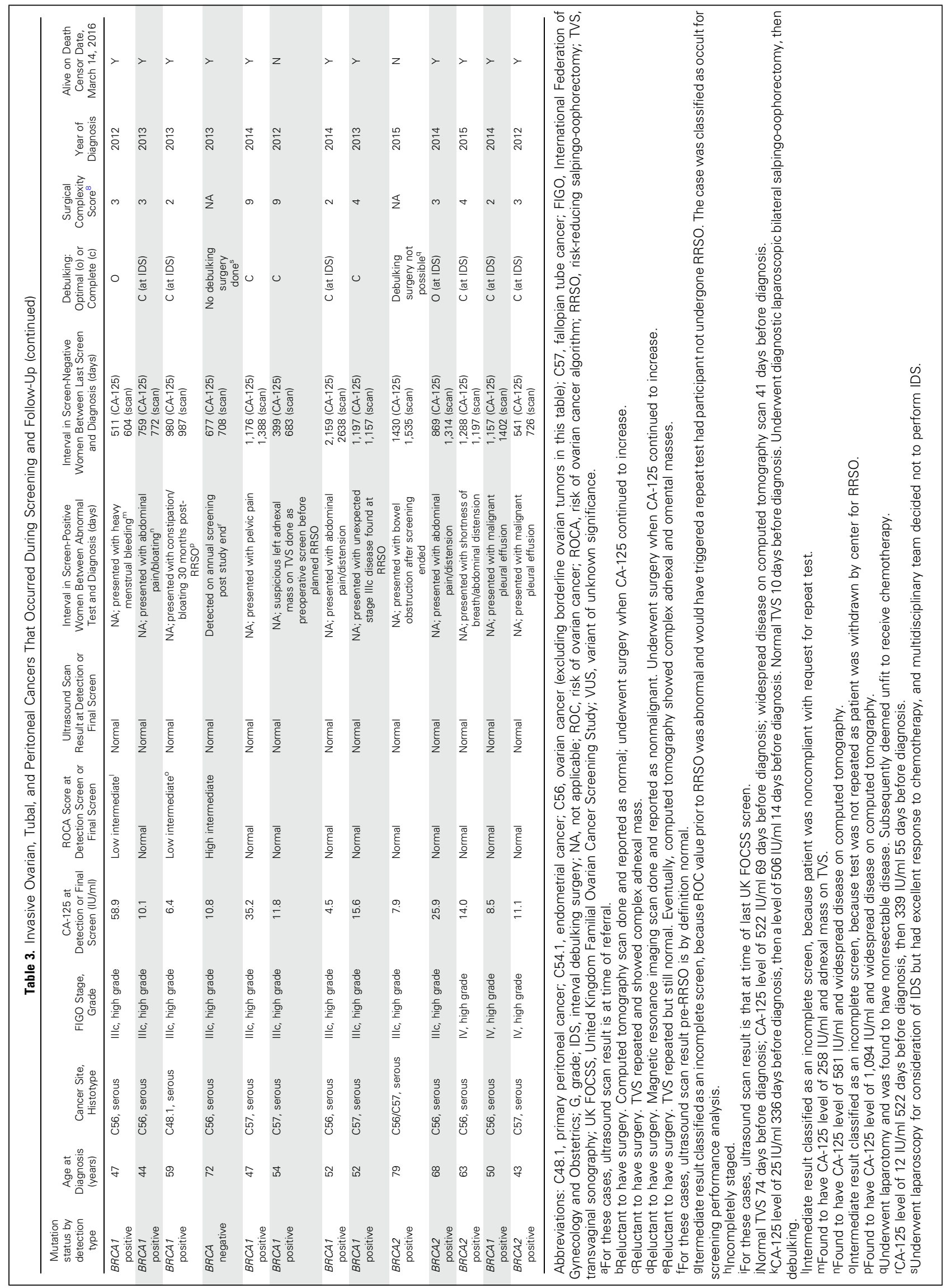


(eight [44.4\%] of 18 women; CI, $21.5 \%$ to $69.2 \% ; P=.008$ ). The mean surgical complexity score ${ }^{28}$ in women diagnosed during screening or less than 365 days after the final screen was 2.7 compared with 4.3 in those diagnosed subsequently (Mann-Whitney $U$ test, $P=.16$ ).

\section{Screening/Surgical Intervals}

The median delay in incident detection screens in this Phase II study was 6 days (range, 0 to 87 days) compared with 88 days (range, 6 to 737 days) in Phase I (gamma generalized linear model, $P=.004)$. The median interval between detection screen and diagnosis in this Phase II study was 82 days (range, 9 to 209 days) compared with 79 days (range, 15 to 184 days) in Phase I ( $P=$ not significant). Reasons for the delay included falsely reassuring scans and reluctance to undergo surgery (Table 3).

\section{Screening Performance}

All 13 cancers (100\%) in women who did not undergo RRSO were screen detected (CI, $75.3 \%$ to $100 \%$ ). Hence, for modeled sensitivity, the lower confidence limit of $75.3 \%$ was used to conservatively estimate the proportion of occult cancers which would have been screen detected had women not undergone RRSO.

Modeled sensitivity, PPV, and negative predictive value (NPV) for the detection of OC/FTC at 1 year for the whole population were $94.7 \%$ (CI, $74.0 \%$ to $99.9 \%$ ), $10.8 \%$ (CI, $6.5 \%$ to $16.5 \%$ ), and $100 \%$ (CI, $100 \%$ to $100 \%$ ), respectively. PPV was significantly better in $B R C A 1 / 2$ carriers than in women who had an unknown mutation status (Table 4).

\section{Comparison of Phase I With Phase II}

Key comparisons of UK FOCSS Phase I and Phase II are listed in Table 5. Rates of clinically presenting interval cancers, zero residual disease after surgery, modeled sensitivity, proportions of women diagnosed with cancer stage less than IIIb, screening delays, and proportions with normal scans at referral were all better in Phase II, but only the comparisons of screening delays and proportions with normal scans at referral were significant.

\section{DISCUSSION}

These UK FOCSS Phase II results suggest that in a high-risk population, ROCA-based multimodal screening every 4 months, alongside reminders of the effectiveness of RRSO, is associated with high sensitivity, significantly lower high-volume disease, and a high zero residual disease rate after surgery compared with women from the same cohort in whom cancer was diagnosed $>1$ year after screening ended. Two similar US studies were published while this paper was in press as a combined analysis, with three of six incident cancers found at stage $\mathrm{I} / \mathrm{II} .^{29}$

The strengths of this study are its size, multicenter setting, centralized screening with a validated algorithm, and reliable multiplesource follow-up. A limitation is the nonrandomized design. However, data about OC diagnosed after screening ended allowed comparisons in the absence of a nonscreening arm. Other limitations include the unknown mutation status of many participants and the small number of incident cancers, which limited power. Although screening delays were effectively eliminated (median, 6 days), the median interval between abnormal results and surgery continued to be $>2$ months (82 days in this Phase II study $v 79$ days in Phase I). There were still some long intervals as a result of patient reluctance to undergo surgery and falsely reassuring imaging associated with an abnormal ROC at referral, as seen in UKCTOCS. ${ }^{23}$

During and within 1 year of UK FOCSS screening, no patients with interval cancers presented with symptoms, sensitivity was

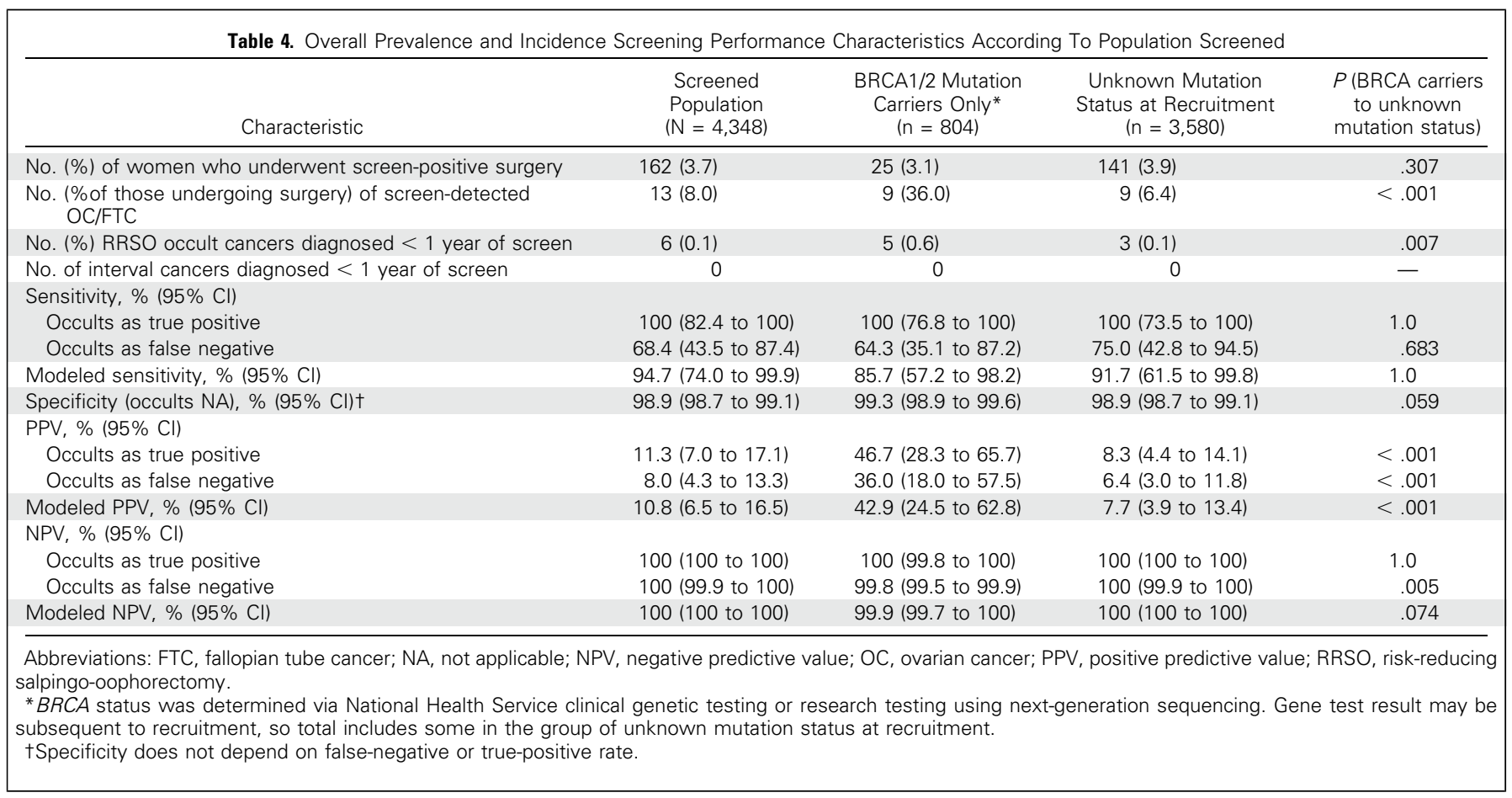


Table 5. Key Comparisons Between UK FOCSS Phase I and Phase II

\begin{tabular}{|c|c|c|c|}
\hline & Phase I & Phase II & \\
\hline Screening Strategy & $\begin{array}{l}\text { Annual CA-125 (with cutoff) } \\
\text { Plus TVS, Arranged Locally }\end{array}$ & $\begin{array}{l}\text { ROCA Every } 4 \text { Months Plus } \\
\text { Annual TVS, Arranged Centrally }\end{array}$ & $P$ \\
\hline Time period & May 6, 2002 to January 1, 2008 & June 14, 2007 to May 15, 2012 & NA \\
\hline No. of participants & 3,563 & 4,348 & NA \\
\hline Women-screen years & 11,366 & 13,728 & NA \\
\hline Median age (range), years & $44.6(35-81)$ & $45.5(34-84)$ & NA \\
\hline No. (\%) of known BRCA1/2 mutation carriers* as a proportion of population & $607(17.0)$ & $804(18.5)$ & NA \\
\hline No. $(\%)$ of occult cancers in known $B R C A$ carriers* who underwent RRSO & $3(1.8)$ in 169 & $9(4.0)$ in 227 & .249 \\
\hline $\begin{array}{l}\text { No. (\%) of incident screen-detected cancers diagnosed at stage IIla or } \\
\text { earlier }\end{array}$ & $5(38.5)$ in 13 & $6(50.0)$ in 12 & .695 \\
\hline Modelled incident sensitivity, (\%) & 82.4 & 88.9 & .658 \\
\hline No. (\%) of incident screen-detected cancers that had zero residual disease & $8(61.5)$ in 13 & $11(91.7)$ in 12 & .160 \\
\hline $\begin{array}{l}\text { No. (\%) of cancers that had zero residual disease }<365 \text { days after last } \\
\text { screen }\end{array}$ & 19 (70.4) in 27 & 18/19 (94.7\%) & .061 \\
\hline Proportion of incident cancers presenting clinically & 2 of $18(n=1$, stage $I ; n=1$, stage IV) & 0 of 18 & .486 \\
\hline $\begin{array}{l}\text { Proportion (\%) of incident screen-detected cancers with a normal scan at } \\
\text { referral }\end{array}$ & 0 of $13(0)$ & 5 of $12(41.7)$ & .015 \\
\hline Median incident screen delay prior to detection (range), days & $88(6-737)$ & $6(0-87)$ & $.004 \dagger$ \\
\hline Median interval between detection screen and surgery (range), days & 77 (15-184) & $80(9-209)$ & $.460 \dagger$ \\
\hline
\end{tabular}

high, and there was a significant stage shift compared with patients who had cancers diagnosed more than 1 year after screening ended. The significantly higher proportion of cancers diagnosed at stage IIIa or lower (ie, microscopic abdominal disease at worst) during the study was associated with more primary surgery and with higher zero residual disease achieved with less complex surgery. Published complete cytoreduction rates in clinically presenting BRCA1/2 carriers ranged from $28 \%$ to $30 \%{ }^{2,30}$ The overall findings suggest a screening-mediated reduction in disease volume. It is likely this would translate into reduced surgical morbidity and fewer incomplete resections. It remains unknown whether this would improve survival in screened high-risk women. We were unable to analyze survival, because there were only three deaths in the 37 women with invasive OC/FTC/PPCs at censorship on March 14, 2016. Although this is encouraging, medium-term survival of OC in $B R C A 1 / 2$ carriers is better than that of $B R C A 1 / 2$-negative patients. ${ }^{7,8}$

The performance characteristics of screening every 4 months were encouraging; overall incident sensitivity was $94.7 \%$, with occult cancer detection modeled, and PPV was $10.8 \%$ (ie, greater than the suggested $10 \%$ level for general-population screening). ${ }^{16}$ However, PPV is less relevant in high-risk populations for whom RRSO is already recommended as optimal management. As expected, PPV was better in BRCA1/2 carriers (42.9\%) than in women who had an unknown mutation status $(7.7 \%)$ because of the lower cancer incidence in women with an unknown status.

The high compliance with blood tests and TVS suggests that the protocol is feasible and acceptable. However, compliance might not be maintained outside a trial. A parallel psychological study found moderate cancer distress at 1 week in women with abnormal ROCA and/or scan results, which led to higher withdrawal from screening. ${ }^{31}$ However, there was no significant effect on general anxiety and/or depression on return to routine screening or at 9 months.

In conclusion, our protocol achieves encouraging performance characteristics, is associated with a low rate of high-volume disease at primary surgery, and had a high zero residual disease rate at low levels of surgical complexity. RRSO remains the treatment of choice for women at high-risk of OC/FTC. In those not ready or willing to undergo surgery, multimodal screening using ROCA every 4 months and TVS (at an interval determined by the ROCA), with regular discussions about the effectiveness of RRSO, appears to be a better option than symptom awareness alone. Such screening should not be viewed as an alternative to surgery, but it does seem to offer a better chance of avoiding a diagnosis of advanced incompletely resectable OC/FTC in the interim.

\section{AUTHORS' DISCLOSURES OF POTENTIAL CONFLICTS} OF INTEREST

Disclosures provided by the authors are available with this article at jco.org.

\section{AUTHOR CONTRIBUTIONS}

Conception and design: Adam N. Rosenthal, James Mackay, Steven J. Skates, Usha Menon, Ian J. Jacobs

Collection and assembly of data: Adam N. Rosenthal, Lindsay S.M. Fraser, Sue Philpott, Ranjit Manchanda, Philip Badman, Richard Hadwin, Ivana Rizzuto, Elizabeth Benjamin, Naveena Singh,

D. Gareth Evans, Diana M. Eccles, Andy Ryan, Robert Liston, Anne Dawnay, Jeremy Ford, Richard Gunu, James Mackay, Usha Menon, Ian J. Jacobs

Data analysis and interpretation: Adam N. Rosenthal, Lindsay S.M. Fraser, Sue Philpott, Ranjit Manchanda, Matthew Burnell, Richard Hadwin, Ivana Rizzuto, Elizabeth Benjamin, Naveena Singh, D. Gareth Evans, Diana M. Eccles, James Mackay, Steven J. Skates, Usha Menon, Ian J. Jacobs

Manuscript writing: All authors

Final approval of manuscript: All authors

Accountable for all aspects of the work: All authors 


\section{REFERENCES}

1. Rubin $\mathrm{SC}$, Blackwood MA, Bandera $\mathrm{C}$, et al: $B R C A 1, B R C A 2$, and hereditary nonpolyposis colorectal cancer gene mutations in an unselected ovarian cancer population: Relationship to family history and implications for genetic testing. Am J Obstet Gynecol 178:670-677, 1998

2. Alsop K, Fereday S, Meldrum C, et al: BRCA mutation frequency and patterns of treatment response in BRCA mutation-positive women with ovarian cancer: A report from the Australian Ovarian Cancer Study Group. J Clin Oncol 30:2654-2663, 2012

3. Antoniou A, Pharoah PD, Narod S, et al: Average risks of breast and ovarian cancer associated with $B R C A 1$ or BRCA2 mutations detected in case series unselected for family history: A combined analysis of 22 studies. Am J Hum Genet 72:1117-1130, 2003

4. Evans DG, Shenton A, Woodward E, et al: Penetrance estimates for BRCA1 and BRCA2 based on ge netic testing in a clinical cancer genetics service setting: Risks of breast/ovarian cancer quoted should reflect the cancer burden in the family. BMC Cancer 8:155, 2008

5. Ramus SJ, Song H, Dicks E, et al: Germline mutations in the BRIP1, BARD1, PALB2, and NBN genes in women with ovarian cancer. J Natl Cancer Inst 107:djv214, 2015

6. Song H, Dicks E, Ramus SJ, et al: Contribution of germline mutations in the RAD51B, RAD51C, and $R A D 51 D$ genes to ovarian cancer in the population J Clin Oncol 33:2901-2907, 2015

7. Chetrit A, Hirsh-Yechezkel G, Ben-David $Y$, et al: Effect of BRCA1/2 mutations on long-term survival of patients with invasive ovarian cancer: The national Israeli study of ovarian cancer. J Clin Oncol 26:20-25, 2008

8. Candido-dos-Reis FJ, Song $\mathrm{H}$, Goode EL, et al: Germline mutation in BRCA1 or BRCA2 and ten-year survival for women diagnosed with epithelial ovarian cancer. Clin Cancer Res 21:652-657, 2015

9. Marchetti C, De Felice F, Palaia I, et al: Riskreducing salpingo-oophorectomy: A meta-analysis on impact on ovarian cancer risk and all cause mortality in BRCA 1 and BRCA 2 mutation carriers. BMC Womens Health 14:150, 2014
10. Finch AP, Lubinski J, Møller $P$, et al: Impact of oophorectomy on cancer incidence and mortality in women with a BRCA1 or BRCA2 mutation. J Clin Oncol 32:1547-1553, 2014

11. Domchek $S M$, Friebel $T M$, Singer $C F$, et al: Association of risk-reducing surgery in BRCA1 or $B R C A 2$ mutation carriers with cancer risk and mortality. JAMA 304:967-975, 2010

12. Michelsen TM, Pripp $A H$, Tonstad $S$, et al: Metabolic syndrome after risk-reducing salpingooophorectomy in women at high risk for hereditary breast ovarian cancer: A controlled observational study. Eur J Cancer 45:82-89, 2009

13. Tuppurainen $M$, Kröger $H$, Honkanen $R$, et al: Risks of perimenopausal fractures: A prospective population-based study. Acta Obstet Gynecol Scand 74:624-628, 1995

14. Ryan J, Scali J, Carrière I, et al: Impact of a premature menopause on cognitive function in later life. BJOG 121:1729-1739, 2014

15. Jacobs IJ, Skates SJ, MacDonald N, et al: Screening for ovarian cancer: A pilot randomised controlled trial. Lancet 353:1207-1210, 1999

16. Rosenthal $A N$, Fraser $L$, Manchanda $R$, et al: Results of annual screening in phase I of the United Kingdom familial ovarian cancer screening study highlight the need for strict adherence to screening schedule. J Clin Oncol 31:49-57, 2013

17. Hogg R, Friedlander M: Biology of epithelia ovarian cancer: Implications for screening women at high genetic risk. J Clin Oncol 22:1315-1327, 2004

18. Stirling D, Evans DG, Pichert $G$, et al: Screening for familial ovarian cancer: Failure of current protocols to detect ovarian cancer at an early stage according to the International Federation of Gynecology and Obstetrics system. J Clin Oncol 23:5588-5596, 2005

19. Vasen HF, Tesfay $E$, Boonstra $H$, et al: Early detection of breast and ovarian cancer in families with BRCA mutations. Eur J Cancer 41:549-554, 2005

20. Hermsen BB, Olivier RI, Verheijen $\mathrm{RH}$, et al: No efficacy of annual gynaecological screening in BRCA1/2 mutation carriers: An observational followup study. Br J Cancer 96:1335-1342, 2007

21. Evans DG, Gaarenstroom KN, Stirling $D$, et al: Screening for familial ovarian cancer: Poor survival of BRCA1/2 related cancers. J Med Genet 46:593-597, 2009
22. Menon U, Gentry-Maharaj $A$, Hallett $R$, et al: Sensitivity and specificity of multimodal and ultrasound screening for ovarian cancer, and stage distribution of detected cancers: Results of the prevalence screen of the UK Collaborative Trial of Ovarian Cancer Screening (UKCTOCS). Lancet Oncol 10:327-340, 2009

23. Menon $U$, Ryan $A$, Kalsi J, et al: Risk algorithm using serial biomarker measurements doubles the number of screen-detected cancers compared with a single-threshold rule in the United Kingdom Collaborative Trial of Ovarian Cancer Screening. J Clin Oncol 33:2062-2071, 2015

24. Jacobs IJ, Menon U, Ryan A, et al: Ovarian cancer screening and mortality in the UK Collaborative Trial of Ovarian Cancer Screening (UKCTOCS): A randomised controlled trial. Lancet 387:945-956, 2016

25. UK Familial Ovarian Cancer Screening Study: http://www.instituteforwomenshealth.ucl.ac.uk/ womens-cancer/gcrc/ukfocss/UKFOCSS Protocol P2_v8_Oct_10_Website_20160525.pdf

26. Widschwendter $M$, Rosenthal $A N$, Philpott $S$ et al: The sex hormone system in carriers of BRCA $1 / 2$ mutations: A case-control study. Lancet Oncol 14: 1226-1232, 2013

27. Skates SJ, Mai $P$, Horick NK, et al: Large prospective study of ovarian cancer screening in high-risk women: CA125 cut-point defined by menopausal status. Cancer Prev Res (Phila) 4:1401-1408, 201

28. Aletti GD, Dowdy SC, Podratz KC, et al: Relationship among surgical complexity, short-term morbidity, and overall survival in primary surgeny for advanced ovarian cancer. Am J Obstet Gynecol 197:676.e1-676.e7, 2007

29. Skates SJ, Greene MH, Buys SS, et al: Early de tection of ovarian cancer using the risk of ovarian cancer algorithm with frequent CA125 testing in women at increased familial risk: Combined results from two screening trials. Clin Cancer Res [Epub ahead of print January 31, 2017]

30. Hyman DM, Long KC, Tanner EJ, et al: Outcomes of primary surgical cytoreduction in patients with BRCA-associated high-grade serous ovarian carcinoma. Gynecol Oncol 126:224-228, 2012

31. Brain KE, Lifford KJ, Fraser $L$, et al: Psychological outcomes of familial ovarian cancer screening: No evidence of long-term harm. Gynecol Oncol 127: 556-563, 2012

\section{Affiliations}

Adam N. Rosenthal, Lindsay S.M. Fraser, Susan Philpott, Ranjit Manchanda, Matthew Burnell, Philip Badman, Richard Hadwin, Ivana Rizzuto, Andy Ryan, Robert Liston, Jeremy Ford, Richard Gunu, Usha Menon, and Ian J. Jacobs, University College London Elizabeth Garrett Anderson Institute for Women's Health; Elizabeth Benjamin, University College London; Naveena Singh, Barts Health National Health Service Trust; Ranjit Manchanda, Barts Cancer Institute, Queen Mary University of London; Anne Dawnay, University College London Hospital; James Mackay, The University College London Cancer Institute, London; D. Gareth Evans, University of Manchester, St Mary's Hospital Manchester, Manchester; Diana M. Eccles, Southampton General Hospital, Southampton, United Kingdom; Steven J. Skates, Massachusetts General Hospital and Harvard Medical School, Boston, MA; and Ian J. Jacobs, University of New South Wales Australia, Sydney, New South Wales, Australia.

\section{Support}

Supported by Cancer Research UK (C1005/A6383, C1005/A7749), The Eve Appeal, and the United Kingdom National Institute for Health Research (NIHR). Researchers at University College London were supported by the NIHR Biomedical Research Centre at University College London Hospitals National Health Service Foundation Trust and University College London.

\section{Prior Presentation}

Previously presented in part at the Annual Meeting of the American Society of Clinical Oncology, Chicago, IL, May 31-June 4, 2013; the British Gynecological Cancer Society Annual Meeting, Belfast, Northern Ireland, June 20-21, 2013; and the 18th Annual Meeting of the European Society of Gynecological Oncology, Liverpool, United Kingdom, October 19-22, 2013. 


\section{AUTHORS' DISCLOSURES OF POTENTIAL CONFLICTS OF INTEREST}

Evidence of Stage Shift in Women Diagnosed With Ovarian Cancer During Phase II of the United Kingdom Familial Ovarian Cancer Screening Study

The following represents disclosure information provided by authors of this manuscript. All relationships are considered compensated. Relationships are self-held unless noted. I = Immediate Family Member, Inst = My Institution. Relationships may not relate to the subject matter of this manuscript. For more information about ASCO's conflict of interest policy, please refer to www.asco.org/rwc or ascopubs.org/jco/site/ifc.

Adam N. Rosenthal

Honoraria: Roche

Consulting or Advisory Role: Myriad Genetics, Abcodia

Speakers' Bureau: Roche

Travel, Accommodations, Expenses: Roche

Lindsay S.M. Fraser

No relationship to disclose

Sue Philpott

No relationship to disclose

Ranjit Manchanda

Other Relationship: Barts and the London Charity; The Eve Appeal; Cancer Research UK; Israel National Institute for Health Policy Research

\section{Matthew Burnell}

No relationship to disclose

\section{Philip Badman}

No relationship to disclose

\section{Richard Hadwin}

No relationship to disclose

\section{Ivana Rizzuto}

No relationship to disclose

Elizabeth Benjamin

No relationship to disclose

\section{Naveena Singh}

No relationship to disclose

D. Gareth Evans

Honoraria: AstraZeneca

Diana M. Eccles

Consulting or Advisory Role: AstraZeneca

Andy Ryan

No relationship to disclose

\section{Robert Liston}

No relationship to disclose

Anne Dawnay

Consulting or Advisory Role: Abcodia

Travel, Accommodations, Expenses: Roche

Jeremy Ford

No relationship to disclose

Richard Gunu

No relationship to disclose

James Mackay

Employment: Myriad Genetics, CIS Oncology

Honoraria: Myriad Genetics International

Consulting or Advisory Role: Company: Myriad Genetics, Abcodia, CIS Oncology

Steven J. Skates

Stock or Other Ownership: SISCAPA Assay Technologies

Consulting or Advisory Role: Abcodia

Patents, Royalties, Other Intellectual Property: Abcodia

Usha Menon

Stock or Other Ownership: Abcodia

Research Funding: Abcodia (Inst)

Patents, Royalties, Other Intellectual Property: European patent No. EP10178345.4: Breast Cancer Diagnostics, which describes the implications of receptor activator of nuclear factor $\mathrm{\kappa}$-B ligand inhibition in the prevention of hormone-driven breast cancer.

Ian J. Jacobs

Employment: Women's Health Specialists

Leadership: Abcodia

Stock or Other Ownership: Abcodia

Consulting or Advisory Role: Abcodia

Patents, Royalties, Other Intellectual Property: ROC Algorithm 


\section{Acknowledgment}

We thank the participants, without whom the study would not have been possible. We thank the lead clinicians at collaborating centers and their research, administrative, and sonography colleagues for their hard work and support over so many years. We thank the independent data monitoring committee (Max Parmar [chair], Shehla Mohammed, Karina Reynolds) and trial steering committee (Julietta Patnick [chair], Diana Eccles, Stephen Duffy, Derek Cruikshank, Louise Bayne). We also thank departmental colleagues Lisa Hinton, Michelle Johnson, Sarah Chamberlain, Jessica Mozersky, Lisa Perreault, Lesley Hague, and Tracy Pearmain and the laboratory team. 